\title{
A SIMPLE DETERMINISTIC MODEL FOR VOLCANIC ASHFALL DEPOSITION
}

\author{
LENG LENG LIM ${ }^{1}$, WINSTON L. SWEATMAN ${ }^{\otimes 1}$ and ROBERT MCKIBBIN ${ }^{1}$
}

(Received 5 May, 2006; revised 27 February, 2008)

\begin{abstract}
We describe a simple deterministic model for the dispersion of particulate ash which has been ejected into the atmosphere by a volcanic eruption. In our model the atmosphere is divided into a series of horizontal layers within which the physical parameters involved are constant. This is an effective way to allow for the changing behaviour of the particulate ash and atmospheric flow with height whilst retaining simplicity. From our model we construct an analytical expression for the final deposit which could be incorporated within hazard assessment projections. In particular we show how to allow for variation with height of dispersion (caused by turbulence due to the wind) and settling speed (affected by the agglomeration and fragmentation of particles).
\end{abstract}

2000 Mathematics subject classification: primary 86A04; secondary 76R50.

Keywords and phrases: eruption, dispersion, environment.

\section{Introduction}

An erupting volcano ejects rock fragments into the atmosphere and further fragmentation produces small ash particles. This volcanic ash can travel hundreds to thousands of kilometres, the distance travelled depending on its size, the strength of the eruption and the physical condition of the atmosphere during the eruption. The falling ash is a hazard within the air, posing a threat to aircraft, and it may disrupt electricity and telephone networks as well as causing destruction and pollution when it settles upon the ground [3]. Models of this dispersion process, both for predicting atmospheric concentrations of ash and for predicting the eventual deposits upon the ground (to produce so-called hazard maps), form an important part of hazard planning and risk evaluation.

Here we describe a simple deterministic model combining advection and dispersion and capturing some of the main physical processes following the eruption. Using this model we find formulae for the atmospheric concentrations and final depositions.

\footnotetext{
${ }^{1}$ Institute of Information and Mathematical Sciences, Massey University at Albany, Private Bag 102 904, North Shore 0745, New Zealand; e-mail: w.sweatman@massey.ac.nz.

(C) Australian Mathematical Society 2008, Serial-fee code 0334-2700/08
} 
With these formulae, it is easy to see the relative importance and the effect of the different physical processes. We can also readily use the formulae to predict what would happen for different atmospheric and particulate conditions.

There has been a long history of modelling atmospheric diffusion of both buoyant and heavier-than-air materials, for example [5, 13-16, 18, 19] and the references in $[20,21]$. Expressions for concentration containing Gaussian functions arise in many of these earlier models.

In this paper, we consider particles which are heavier than air and which progressively move downwards towards the ground. The descent is very rapid for large particles. We assume that, when particles reach a particular height, there is a dominant length scale for their dispersion. Different constants may be used with different size particles or different times or heights of release. Stratification of the atmosphere into layers enables this to be done. Gaussian expressions arise from our model.

In our previous work [12], we have considered several aspects of the overall dispersion process. Here we focus upon the later stages of the ashfall and in particular provide expressions predicting the form of the deposit upon the ground. Other work, also using layered models, has considered the effect upon such deposits of the variation in wind velocity with height [1]. However, our model also includes variation with height of the dispersion due to turbulence (which is to be expected with an elevation-dependent wind speed) and further we model the effect of fragmentation or agglomeration of ash particles by allowing the settling speed to vary with height.

The model we present is simple. It is complementary to the more massive fluid dynamical numerical simulations that are also used to model such events. Our aim is to capture and understand essential features of the dispersion. However, by combining different cohorts of particles, with different sizes, release heights or release times, our model may be readily built up from components when necessary to model a more complicated release.

\section{The model}

2.1. The layered atmosphere For the kind of volcanic eruption considered here, particles are ejected into the atmosphere and thereafter these fall under their own gravity, whilst being blown along by the wind [6]. The pattern and distribution of the ash are highly dependent upon the physical conditions of the atmosphere during the ashfall. The atmosphere is modelled as a sequence of uniform horizontal layers in the half-space $(-\infty<x, y<\infty, 0 \leq z<\infty)$. This is more realistic than a uniform atmosphere as it allows for the variation of parameters with elevation. Each layer interface corresponds to a change in atmospheric conditions such as wind speed, wind direction or dominant turbulent length scale (these might be sourced from meteorological data). We number the layers downwards from 1, in which the release occurs, to $n$, immediately above the ground. The interface boundary between the $j$ th and $(j+1)$ th layers occurs at height $z=Z_{j}$. We have assumed that the variation of topography on the ground itself $\left(z=Z_{n}=0\right)$ is not severe enough to influence the average transport mechanisms. 
The effect of the initial eruption is to transport the small particles of ash to a height $H$ above the volcano. Falling downwards, such particles rapidly reach the settling speed $S$, which is the terminal speed at which the force due to gravity is exactly balanced by the drag. The particles will also rapidly gather momentum to move along with the wind. For the purposes of our model, it is a reasonable approximation to assume that the particles, both at release and thereafter, are falling at their settling speed and are moving with the same velocity as the wind [12].

We consider an instantaneous release of volcanic ash into the atmosphere at the point $(x, y, z)=\left(X_{0}, Y_{0}, H\right)$ at time $t=t_{0}$. The total mass is taken to be $Q$ and the particles are taken to be uniform in size. The release is into the top layer of our layered atmosphere and so $Z_{1} \leq H<\infty$. In Section 3 we show how the effects of more complicated volcanic eruptions may be captured by summation over a number of such distributions with varying release points, particle sizes or release times.

The particles are transported by the wind with a velocity $\mathbf{u}=(U, V, 0)$, which in general varies in both speed and direction with height. We assume that the wind is strictly horizontal and only dependent upon height. The components $U$ and $V$ are taken to be constant within each horizontal layer, with values $U_{j}$ and $V_{j}$ in the $j$ th layer.

The turbulence, which is caused by the wind, also varies with height and in part this can be attributed to differing wind speeds. At a given height, turbulence within the air flow is modelled as having a certain characteristic length-since turbulence has a variety of scales, the length is a typical dominant mean value for the flow. The effect of air turbulence is incorporated using a dispersion tensor $\underline{\mathbf{D}}$ whose components are constant within each layer.

Experimental observations of turbulence have suggested that the effective dispersion tensor changes with the scale of the dispersing plume. This is not allowed for by Gaussian dispersion in its simplest form. In particular in an early paper Sutton [16] attempts to model dispersion using an empirical formula wherein the effective dispersion rate is given by a fractional power of the distance travelled. Essentially, by implication, this will mean having a dominant length scale which evolves with time. Pasquill [13] also comments that it is not unreasonable to assume that locally, with respect to height and time, there is a near-steady homogeneous structure. His model also allows for a change in length scale with time due to the spread of the release. Such effects are not excluded from our model. Our particle release falls downwards and so, as we vary the dispersion length scale and velocity constants with height, we can also effectively allow for changes with time after release. Releases at differing heights may also be included in such models by linearly combining different cohorts of particles as described in Section 3.

During the process of ashfall the size of the particles may further change. In humid regions of the atmosphere, water droplets may link up neighbouring particles, which then agglomerate into larger particles with a larger settling speed. Alternatively, further fragmentation of particles would create smaller particles. These processes will affect the settling speed $S$ of the ash and so we build variation in this also into our model. 


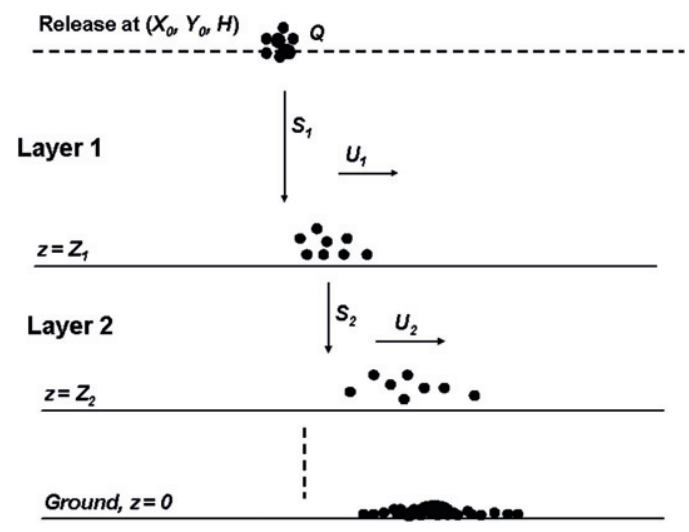

FIGURE 1. A schematic diagram to illustrate the deposition of particles through a layered atmosphere.

We allow settling speed to vary with height, taking a constant value $S_{j}$ in the $j$ th layer. As for the initial release, we assume that the new settling speeds are effectively achieved very quickly.

In practice any predictions will be limited by the inherent variability between similar releases due to turbulent dispersion. The models in this paper aim to recreate a typical event (variability of this kind has been explored in [17]).

Figure 1 illustrates our model for the atmosphere. For each uniform horizontal layer there may be a different wind speed, wind direction, dispersion rate and settling speed.

2.2. The advection-dispersion process The advection-dispersion equation is derived from conservation of mass and is a well-known approach for describing the transport of particles by wind and scattering by dispersion (see for example [21]). We shall derive a form suitable for modelling volcanic ashfall [12].

The advection-dispersion equation is

$$
\frac{\partial c}{\partial t}=-\nabla \cdot \mathbf{q}+m,
$$

where $c$ is the mass concentration of the particles (mass per unit volume of atmosphere), $m$ is a source mass rate (this is, due to new particles introduced into the atmosphere by the volcano and is in units of mass per unit time per unit volume) and $\mathbf{q}$ is mass flux of particles per unit area (the movement of particles through the atmosphere). This latter quantity $\mathbf{q}$ is composed of three distinct components:

$$
\mathbf{q}=c \mathbf{u}-c S \mathbf{k}-\underline{\mathbf{D}} \nabla c .
$$


The advective flux $c \mathbf{u}$ is due to transport by the wind, while $-c S \mathbf{k}$ is the advective flux due to the settling of the particles by gravity $(\mathbf{k}=(0,0,1)$ is the unit vector upwards). The (mechanical) dispersive flux $-\underline{\mathbf{D}} \nabla c$ is caused by the atmospheric turbulence.

The dispersion due to turbulence is assumed to be the same in the crosswind direction as in the downwind direction. Previous models by volcanologists $[1,4,8]$ have also made this assumption. Under these conditions the dispersion tensor

$$
\underline{\mathbf{D}}=\operatorname{diag}\left(D_{x}, D_{y}, D_{z}\right)
$$

will be isotropic in the $x y$-plane, that is $D_{x}=D_{y}=D_{h}$.

The dispersion tensor is often taken to be directly proportional to the wind speed. If we choose axes aligned with the wind, the tensor then has the form $\underline{\mathbf{D}}=|\mathbf{u}| \underline{\mathbf{L}}$, where $\underline{\mathbf{L}}$ is a diagonal dispersion length tensor whose elements are the dominant length scales of atmospheric turbulence. For this form of the dispersion, if there is no wind then there is no dispersion and the particles settle vertically to the ground. For the present, we will not explicitly express the dispersion as a function of the wind velocity, but we shall take it to be constant as well as horizontally isotropic in each of our layers. In layer $j$ the constant value of the horizontal dispersion, $D_{h}$, is denoted $D_{h j}$.

Several authors have noted that the vertical dispersion, $D_{z}$, is very small at heights of $500 \mathrm{~m}$ and above and can be neglected, for example [1, 2, 7]. To further simplify our model, we too will assume that the vertical dispersion is zero.

From (2.1) and (2.2), we obtain

$$
\frac{\partial c}{\partial t}+\nabla \cdot(c \mathbf{u}-c S \mathbf{k}-\underline{\mathbf{D}} \nabla c)=m .
$$

With our assumptions, in the $j$ th layer this equation becomes

$$
\begin{aligned}
\frac{\partial c}{\partial t}+ & U_{j} \frac{\partial c}{\partial x}+V_{j} \frac{\partial c}{\partial y}-S_{j} \frac{\partial c}{\partial z}-D_{h j} \frac{\partial^{2} c}{\partial x^{2}}-D_{h j} \frac{\partial^{2} c}{\partial y^{2}} \\
& =Q \delta\left(x-X_{0}\right) \delta\left(y-Y_{0}\right) \delta(z-H) \delta\left(t-t_{0}\right),
\end{aligned}
$$

where $Z_{j}<z<Z_{j-1}$. As $D_{z}=0$, the downward flux $S c+D_{z}(\partial c / \partial z)$ is just $S c$. We assume that all of the source material lands on the ground eventually and the downward flux is continuous through each interface. We have the initial condition

$$
c(x, y, z, t)=0
$$

when $t=t_{0}$ except at $(x, y, z)=\left(X_{0}, Y_{0}, H\right)$. The boundary conditions are $c(x, y, z, t)=0$ when $x \rightarrow \pm \infty, y \rightarrow \pm \infty$ or $z \rightarrow+\infty$, and $D_{z}(\partial c / \partial z)=0$ on the ground $z=Z_{n}=0$ (there is no dispersion through the ground). However, since $D_{z}=0$, the latter condition is automatically satisfied.

2.3. Concentration and deposition As the $z$-component of dispersion is zero $\left(D_{z}=0\right)$, the vertical motion of the particles through the atmosphere is strictly downwards. For an instantaneous point release, the particles are transported 
horizontally both with the wind and by dispersion. However, vertically they move downwards as a horizontal sheet, with the local settling speed, to eventually strike the ground simultaneously. The lower layers have no influence upon the dynamics above them: at a given height the particles' distribution is solely dependent upon what has happened to them at earlier times, or higher positions, in their fall.

The time $t_{j}$ for which the horizontal sheet of particles reaches height $Z_{j}$ at the interface between layer $j$ and layer $j+1$ is given by

$$
t_{1}=t_{0}+\frac{H-Z_{1}}{S_{1}} \quad \text { and } \quad t_{j}=t_{j-1}+\frac{Z_{j-1}-Z_{j}}{S_{j}} \text { for } j \geq 2 .
$$

With a single layer $(n=1)$ the concentration is $[9,11]$

$$
\begin{aligned}
c(x, y, z, t)= & \frac{Q \delta\left(z-\left(H-S_{1}\left(t-t_{0}\right)\right)\right)}{4 \pi D_{h 1}\left(t-t_{0}\right)} \\
& \times \exp \left[-\frac{\left(x-X_{0}-U_{1}\left(t-t_{0}\right)\right)^{2}}{4 D_{h 1}\left(t-t_{0}\right)}-\frac{\left(y-Y_{0}-V_{1}\left(t-t_{0}\right)\right)^{2}}{4 D_{h 1}\left(t-t_{0}\right)}\right],
\end{aligned}
$$

and the deposit upon the ground $f(x, y)$ (mass per unit area at point $(x, y, 0))$ is obtained by integration with respect to time:

$$
\begin{aligned}
f(x, y)= & \frac{S_{1} Q}{4 \pi D_{h 1} H} \exp \left(-\left[\left(x-\left(X_{0}+U\left(H / S_{1}\right)\right)\right)^{2} /\left(4 D_{h 1}\left(H / S_{1}\right)\right)\right]\right. \\
& \left.-\left[\left(y-\left(Y_{0}+V_{1}\left(H / S_{1}\right)\right)\right)^{2} /\left(4 D_{h 1}\left(H / S_{1}\right)\right)\right]\right) .
\end{aligned}
$$

From this result, the solution with multiple layers may be constructed one layer at a time. Let us illustrate using two layers. The ash is released in the top layer and whilst falling as a sheet through this layer the concentration is exactly the same as for a single layer as given above. Upon reaching the interface between the layers, at height $z=Z_{1}$ and time $t=t_{1}$, the distribution is given by

$$
\begin{aligned}
c\left(x, y, z, t_{1}\right)= & \frac{Q \delta\left(z-Z_{1}\right)}{4 \pi D_{h 1}\left(t_{1}-t_{0}\right)} \exp \left(-\left[\left(x-X_{0}-U_{1}\left(t_{1}-t_{0}\right)\right)^{2} /\left(4 D_{h 1}\left(t_{1}-t_{0}\right)\right)\right]\right. \\
& \left.-\left[\left(y-Y_{0}-V_{1}\left(t_{1}-t_{0}\right)\right)^{2} /\left(4 D_{h 1}\left(t_{1}-t_{0}\right)\right)\right]\right) .
\end{aligned}
$$

Now consider a small element of this distribution at point $(x, y, z)=\left(\xi, \eta, Z_{1}\right)$ on the interface surface:

$$
\begin{aligned}
& q_{1}(\xi, \eta) d \xi d \eta \\
&= c\left(\xi, \eta, Z_{1}, t_{1}\right) d \xi d \eta \\
&= \frac{Q}{4 \pi D_{h 1}\left(t_{1}-t_{0}\right)} \\
& \quad \times \exp \left[\frac{-\left(\xi-X_{0}-U_{1}\left(t_{1}-t_{0}\right)\right)^{2}-\left(\eta-Y_{0}-V_{1}\left(t_{1}-t_{0}\right)\right)^{2}}{4 D_{h 1}\left(t_{1}-t_{0}\right)}\right] d \xi d \eta
\end{aligned}
$$


This behaves like a point source for the second layer. Computing the concentration corresponding to this source element for layer 2 and then integrating over the entire interface surface, we have, for $t_{1}<t<t_{2}$,

$$
\begin{aligned}
c(x, y, z, t)= & \int_{-\infty}^{\infty} \int_{-\infty}^{\infty} \frac{Q}{4 \pi D_{h 1}\left(t_{1}-t_{0}\right)} \frac{\delta\left(z-\left(Z_{1}-S_{2}\left(t-t_{1}\right)\right)\right)}{4 D_{h 2}\left(t-t_{1}\right)} \\
& \times \exp \left[\frac{-\left(\xi-X_{0}+U_{1}\left(t_{1}-t_{0}\right)\right)^{2}-\left(\eta-Y_{0}-V_{1}\left(t_{1}-t_{0}\right)\right)^{2}}{4 D_{h 1}\left(t_{1}-t_{0}\right)}\right] \\
& \times \exp \left[\frac{-\left(x-\xi-U_{2}\left(t-t_{1}\right)\right)^{2}-\left(y-\eta-V_{2}\left(t-t_{1}\right)\right)^{2}}{4 D_{h 2}\left(t-t_{1}\right)}\right] d \xi d \eta \\
= & \frac{Q \delta\left(z-Z_{\mathcal{W}}(t)\right)}{4 \pi \Gamma_{\mathcal{W}}(t)} \exp \left[\frac{-\left(x-X_{\mathcal{W}}(t)\right)^{2}-\left(y-Y_{\mathcal{W}}(t)\right)^{2}}{4 \Gamma_{\mathcal{W}}(t)}\right]
\end{aligned}
$$

where

$$
\begin{aligned}
X_{\mathcal{W}}(t) & =X_{0}+U_{1}\left(t_{1}-t_{0}\right)+U_{2}\left(t-t_{1}\right) \\
Y_{\mathcal{W}}(t) & =Y_{0}+V_{1}\left(t_{1}-t_{0}\right)+V_{2}\left(t-t_{1}\right), \\
Z_{\mathcal{W}}(t) & =H-Z_{1}-S_{2}\left(t-t_{1}\right) \\
& =H-S_{1}\left(t_{1}-t_{0}\right)-S_{2}\left(t-t_{1}\right), \\
\Gamma_{\mathcal{W}}(t) & =D_{h 1}\left(t_{1}-t_{0}\right)+D_{h 2}\left(t-t_{1}\right) .
\end{aligned}
$$

If we repeat this process, introducing further lower layers, we obtain the concentration for the multilayered system. This has the same general form:

$$
\begin{aligned}
c(x, y, z, t)= & \frac{Q}{4 \pi \Gamma_{\mathcal{W}}(t)} \exp \left(-\left[\left(\left(x-X_{\mathcal{W}}(t)\right)^{2} /\left(4 \Gamma_{\mathcal{W}}(t)\right)\right)\right.\right. \\
& \left.\left.+\left(\left(y-Y_{\mathcal{W}}(t)\right)^{2} /\left(4 \Gamma_{\mathcal{W}}(t)\right)\right)\right]\right) \delta\left(z-Z_{\mathcal{W}}(t)\right)
\end{aligned}
$$

for $t_{j-1}<t<t_{j}$ and $Z_{j}<z<Z_{j-1}$, where

$$
\begin{aligned}
X_{\mathcal{W}}(t) & =X_{0}+U_{1}\left(t_{1}-t_{0}\right)+U_{2}\left(t_{2}-t_{1}\right)+\cdots+U_{j}\left(t-t_{j-1}\right), \\
Y_{\mathcal{W}}(t) & =Y_{0}+V_{1}\left(t_{1}-t_{0}\right)+V_{2}\left(t_{2}-t_{1}\right)+\cdots+V_{j}\left(t-t_{j-1}\right), \\
Z_{\mathcal{W}}(t) & =H-S_{1}\left(t_{1}-t_{0}\right)-S_{2}\left(t_{2}-t_{1}\right)-\cdots-S_{j}\left(t-t_{j-1}\right), \\
\Gamma_{\mathcal{W}}(t) & =D_{h 1}\left(t_{1}-t_{0}\right)+D_{h 2}\left(t_{2}-t_{1}\right)+D_{h 3}\left(t_{3}-t_{2}\right)+\cdots+D_{h j}\left(t-t_{j-1}\right) .
\end{aligned}
$$

Again, the deposition is found by integrating the downwards flux at the ground:

$$
\begin{aligned}
f(x, y) & =\int_{0}^{\infty} S_{n} c(x, y, 0, t) d t \\
& =\int_{0}^{\infty} \frac{S_{n} Q}{4 \pi \Gamma_{\mathcal{W}}(t)} \exp \left[-\frac{\left[\left(x-X_{\mathcal{W}}(t)\right)^{2}+\left(y-Y_{\mathcal{W}}(t)\right)^{2}\right]}{4 \Gamma_{\mathcal{W}}(t)}\right] \delta\left(0-Z_{\mathcal{W}}(t)\right) d t \\
& =\frac{Q}{4 \pi \Gamma_{\mathcal{W}}\left(t_{n}\right)} \exp \left[-\frac{\left[\left(x-X_{\mathcal{W}}\left(t_{n}\right)\right)^{2}+\left(y-Y_{\mathcal{W}}\left(t_{n}\right)\right)^{2}\right]}{4 \Gamma_{\mathcal{W}}\left(t_{n}\right)}\right]
\end{aligned}
$$

where $t_{n}$ is the time when the particles land on the ground. 
TABLE 1. Initial data for the release shown in Figure 2.

\begin{tabular}{lcrrrr}
\hline Parameter & & Layer 1 & Layer 2 & Layer 3 & Layer 4 \\
\hline$X_{0}$ & 0 & & & & \\
$Y_{0}$ & 0 & & & & \\
$H$ & 7500 & & & & \\
$Q$ & $25 \times 10^{9}$ & & & & \\
$U$ & & 10 & -10 & 10 & -10 \\
$V$ & & 0 & 0 & 0 & 0 \\
$S$ & & 1 & 1 & 1 & 1 \\
$D_{h}$ & & 800 & 800 & 800 & 800 \\
$D_{z}$ & & 0 & 0 & 0 & 0 \\
\hline
\end{tabular}

The expression for the deposit, the distribution upon the ground of mass per unit area following the eruption, is a simple uncorrelated Gaussian distribution in the two position coordinates $x$ and $y$. As the dispersion is horizontally isotropic, the spread of particles about the centre of mass is the same in all directions. As a result, the isomass contours for this deposit are concentric circles about the centre of mass.

Our solutions for the concentration and deposition have been parameterized in terms of the variables $X_{\mathcal{W}}(t), Y_{\mathcal{W}}(t), Z_{\mathcal{W}}(t)$ and $\Gamma_{\mathcal{W}}(t)$, which are all functions of time. They incorporate the parameters from the separate layers each weighted by the time spent in the layer. The mean horizontal displacement is given by $X_{\mathcal{W}}(t)$ and $Y_{\mathcal{W}}(t)$. The vertical displacement $Z_{\mathcal{W}}(t)$ is the height of the horizontal sheet of falling particles. These are also the coordinates of the position with the highest concentration at time $t$, and the eventual deposition on the ground has a maximum at $\left(X_{\mathcal{W}}\left(t_{n}\right), Y_{\mathcal{W}}\left(t_{n}\right)\right)$

The weighted dispersion effect coefficient $\Gamma_{\mathcal{W}}(t)$ is a measure of the overall effect of the dispersion due to the time spent in the different layers. The spread, and conversely relative density, of the deposit depends only upon this coefficient. A measure of the spread of the final deposit is the standard deviation of the underlying normal distribution, which is $\sqrt{2 \sigma_{\mathcal{W}}\left(t_{n}\right)}$. The maximum value of the mass concentration per unit area in the deposit is $\left(Q /\left(4 \pi \sigma_{\mathcal{W}}\left(t_{n}\right)\right)\right)$. As is to be expected, these measures of the deposit are related, as the total mass is fixed.

Figure 2 illustrates the mass concentration distributions arising from an instantaneous point-source release. For this example we have a four-layered atmosphere. The layer interfaces are at $z=Z_{1}=5000 \mathrm{~m}, z=Z_{2}=3000 \mathrm{~m}$ and $z=Z_{3}=1000 \mathrm{~m}$. (We use SI units for all measurements except where explicitly stated otherwise.) The mass concentration distributions shown at the interfaces may also be interpreted as the deposit which would occur were the ground to be at this height. Other release parameters are given in Table 1. We can see from the figures how the different wind directions in the layers blow the particle distribution along with them. As time is spent falling towards the ground, the spread of the mass concentration becomes progressively wider and consequently the mass 


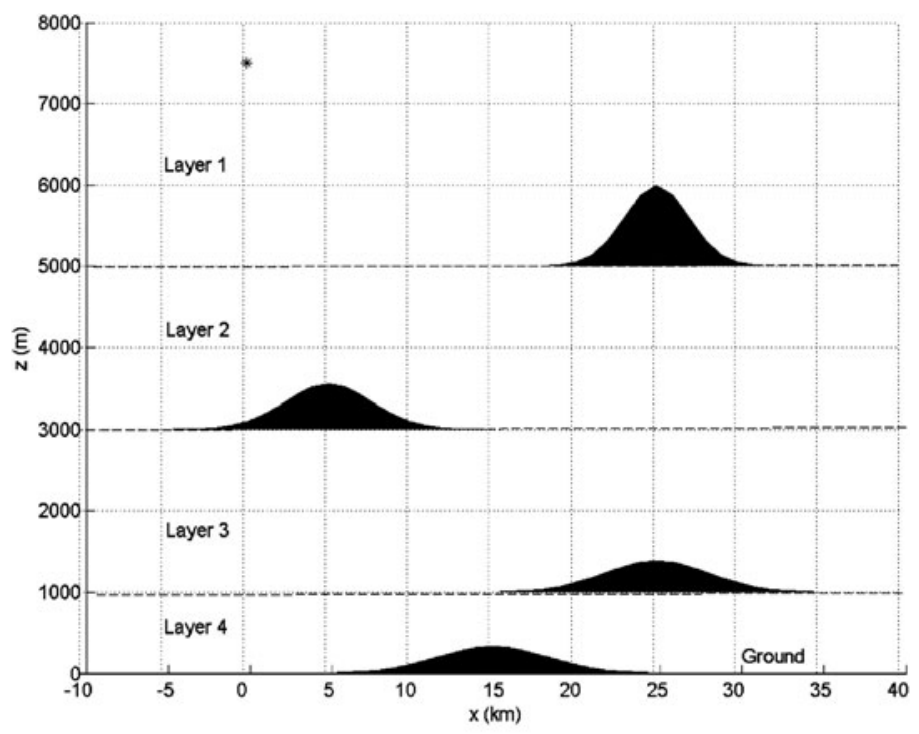

Mass distribution profiles at the layer interfaces and on the ground

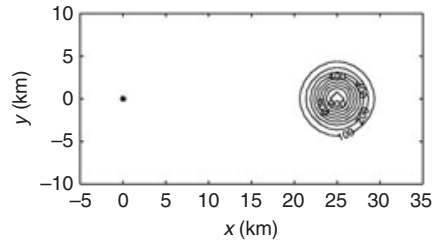

Isomass contours, $z=5000 \mathrm{~m}$

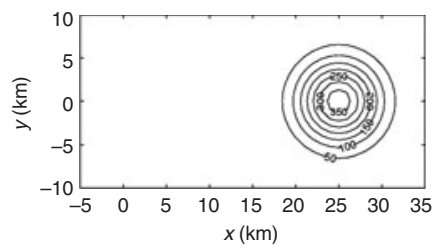

Isomass contours, $z=1000 \mathrm{~m}$

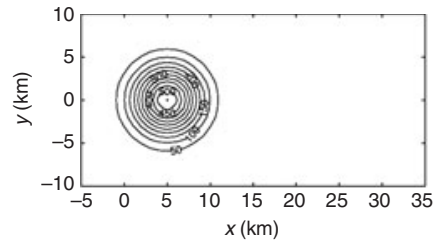

Isomass contours, $z=3000 \mathrm{~m}$

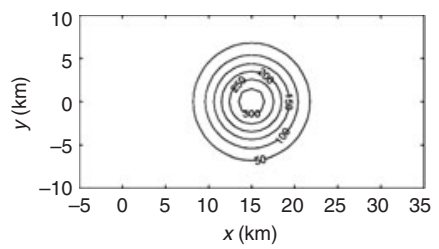

Isomass contours, $z=0 \mathrm{~m}$ (ground)

FIGURE 2. The mass distribution of ashfall for an instantaneous point-source release into a four-layered atmosphere (details are given in the text). The release point, $\left(X_{0}, Y_{0}, H\right)=(0,0,7500) \mathrm{m}$, is marked $*$.

concentration reduces. The figure also illustrates the circularity of isomass contours predicted for an instantaneous point-source release by our model.

\section{Modelling more complicated eruption events}

As the particles in our simple model move and spread independently, we may add together linear combinations of many such instantaneous point releases. By this means we may model the initial conditions, deposition and concentration for a more 
TABLE 2. Layer parameters for the two sources of Figure 3.

\begin{tabular}{llrrr}
\hline Source & Parameter & Layer 1 & Layer 2 & Layer 3 \\
\hline a & $U$ & 10 & -5 & 10 \\
& $V$ & 0 & 0 & 0 \\
& $S$ & 1 & 1 & 1 \\
& $D_{h}$ & 800 & 800 & 800 \\
$\mathrm{~b}$ & $U$ & 10 & 10 & 10 \\
& $V$ & 0 & 0 & 0 \\
& $S$ & 1.5 & 1.5 & 1.5 \\
& $S$ & 800 & 800 & 800 \\
\hline & $D_{h}$ & & & \\
\hline
\end{tabular}
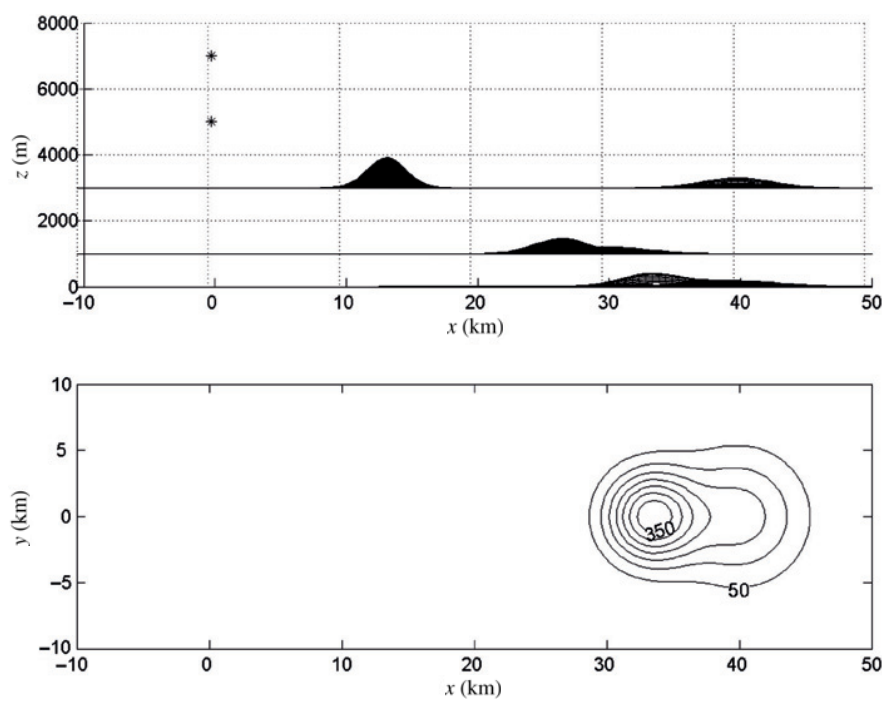

FIGURE 3. The mass distribution of ashfall for a multiple point release into a three-layered atmosphere (details are given in the text). The two point-source release points, $\left(X_{0}, Y_{0}, H\right)=(0,0,7000) \mathrm{m}$ (source a) and $\left(X_{0}, Y_{0}, H\right)=(0,0,5000) \mathrm{m}$ (source b), are marked $*$. In the upper graph the distribution profile is shown at each of the layer interfaces as well as for the eventual deposit on the ground. In the lower graph equally spaced isomass contours are shown for this deposit.

complicated eruption profile. This approach is required to obtain the noncircular deposition contours that occur in reality. Our model contains the parameters $X_{0}$, $Y_{0}, H, Z_{1}, \ldots, Z_{n}, t_{0}, U_{1}, \ldots, U_{n}, V_{1}, \ldots, V_{n}, S_{1}, \ldots, S_{n}, D_{h 1}, \ldots, D_{h n}$. It is these which we can vary and sum or integrate over. For example, we may integrate $\left(X_{0}, Y_{0}, H\right)$ over a range to obtain a dispersed source or integrate $t_{0}$ over a range for a noninstantaneous eruption. Alternatively, by adding results for different values of $S_{j}$, we can include different local settling speeds which would be necessary for a range of particle sizes. Results with different values of $D_{h j}$ can capture changes in the atmospheric conditions or the evolution of the effective dispersion length scale. 
Figure 3 is an illustration of a combined release with two instantaneous point sources. The release points are $\left(X_{0}, Y_{0}, H\right)=(0,0,7000) \mathrm{m}$ for source a and $\left(X_{0}, Y_{0}, H\right)=(0,0,5000) \mathrm{m}$ for source $\mathrm{b}$. The atmosphere has three layers with layer interfaces at $z=3000 \mathrm{~m}$ and $z=1000 \mathrm{~m}$. We have chosen the same combined total mass overall as in the previous example $\left(25 \times 10^{9} \mathrm{~kg}\right)$; however, for this example it is divided equally between the two sources. The layer parameters for this combined release are given in Table 2. It might be easiest to interpret this deposition as the result of two successive eruptions. The layer parameters for source b differ from those for source a for the wind velocity in layer 2 and the settling speed (which could be due to a change in atmospheric humidity). Our resulting deposit is elongated rather than circular.

Elsewhere, we have presented some further examples of linear combinations of point releases [10]. These examples are for a single-layered atmosphere and illustrate how in some conditions different shapes of the source can result in virtually identical deposits. This is an indication of the difficulty presented by the inverse problem to deduce facts about an initial atmospheric release from deposits on the ground.

\section{Conclusions}

We have presented a simple deterministic model for volcanic ashfall which can be used to predict mass concentration in the atmosphere and the final deposition of ash on the ground. By dividing the atmosphere into a number of uniform horizontal layers, we allow the physical conditions, such as wind speed and dispersion rate, to vary with height whilst maintaining the essential simplicity of the model. These horizontal layers enable us to present a more realistic transport model for the atmosphere than would be given by assuming it to be uniform.

The analytical solutions presented for an instantaneous point mass release may be easily combined linearly. By this approach we may produce deposition and atmospheric mass concentration predictions for possibly much more complicated initial conditions arising after a volcanic eruption.

\section{Acknowledgements}

We are grateful to Mark Nelson (University of Wollongong) for his helpful comments and to the referees for their suggestions.

\section{References}

[1] C. Bonadonna, G. Macedonio and R. S. J. Sparks, "Numerical modelling of tephra fallout associated with dome collapses and Vulcanian explosions: application to hazard assessment on Montserrat", in The eruption of Soufriere Hill volcano, Montserrat, from 1995 to 1999 (eds. T. H. Druitt and B. P. Kokelaar), (The Geological Society of London, London, 2002) 517-537.

[2] S. N. Carey, "Modelling of tephra fallout from explosive eruption", in Monitoring and mitigation of volcano hazards (eds. R. Scarpa and R. I. Tilling), (Springer, Berlin, 1996) 429-461.

[3] T. J. Casadevall, "The 1989-1990 eruption of Redoubt Volcano, Alaska: impacts on aircraft operations", J. Volcanol. Geothermal Res. 62 (1994) 301-316. 
[4] C. B. Connor, B. E. Hill, B. Winfrey, N. M. Franklin and P. C. La Femina, "Estimation of volcanic hazards from tephra fallout", Natural Hazards Rev. 2 (2001) 33-42.

[5] G. T. Csanady, "Turbulent diffusion of heavy particles in the atmosphere", J. Atmos. Sci. 20 (1963) 201-208.

[6] R. E. Holasek, A. W. Woods and S. Self, "Experiments on gas-ash separation processes in volcanic umbrella plumes", J. Volcanol. Geothermal Res. 70 (1996) 169-181.

[7] A. W. Hurst, "ASHFALL - a computer program for estimating volcanic ash fallout", Report and user guide, Science Report 94/23, Institute of Geological \& Nuclear Sciences, 1994.

[8] A. W. Hurst and R. Turner, "Performance of the program ASHFALL for forecasting ashfall during the 1995 and 1996 eruptions of Ruapehu volcano", J. Volcanol. Geothermal Res. 76 (1997) 163171.

[9] J. Kevorkian, Partial differential equations: analytical solution techniques (Chapman and Hall, London, 1993).

[10] L. L. Lim, W. L. Sweatman, R. McKibbin and C. B. Connor, "Tephra fallout models: the effect of different source shapes on isomass maps", Math. Geosci. 40 (2008) 147-157.

[11] J. D. Logan, Applied partial differential equations (Springer, Berlin, 1998).

[12] R. McKibbin, L. L. Lim, T. A. Smith and W. L. Sweatman, "A model for dispersal of eruption ejecta", in Proc. World Geothermal Conf. 2005, Antalya, Turkey, 24-29 April 2005 (eds.

R. D. Horne and E. Okandan), (International Geothermal Association, Pisa, 2005).

[13] F. Pasquill, "The estimation of the dispersion of windborne material", Meteorol. Mag. 90 (1961) 33-49.

[14] L. Richardson, "Atmospheric diffusion shown on a distance-neighbour graph", Proc. Roy. Soc. A 110 (1926) 709-737.

[15] O. F. T. Roberts, "The theoretical scattering of smoke in a turbulent atmosphere", Proc. R. Soc. Lond., Ser. A 104 (1923) 640-654.

[16] O. G. A. Sutton, "Theory of eddy diffusion in the atmosphere", Proc. R. Soc. Lond., Ser. A 135 (1932) 143-165.

[17] W. L. Sweatman and P. C. Chatwin, "Dosages from instantaneous releases of dense gases in wind tunnels and into a neutrally stable atmosphere", Boundary-Layer Meteorol. 77 (1996) 211-231.

[18] G. I. Taylor, "Eddy motion in the atmosphere", Philos. Trans. R. Soc. Lond., Ser. A 215 (1915) $1-26$.

[19] G. I. Taylor, "Diffusion by continuous movements", Proc. London Math. Soc. (2) 20 (1922) 196212.

[20] D. B. Turner, Workbook of atmospheric dispersion estimates: an introduction to dispersion modeling, 2nd edn (CRC Press, Boca Raton, FL, 1994).

[21] C. Zheng and G. D. Bennett, Applied contaminant transport modelling (Wiley-Interscience, New York, 2002). 